\title{
REAL POLYNOMIAL FORM OF MUSIC FOR UNIFORM LINEAR ARRAY
}

\author{
Xiang Cao*, Jingmin Xin \\ Xi' an Jiaotong University \\ Institute of Artificial Intelligence and \\ Robotics \\ Xi'an 710049, China
}

\author{
Yoshifumi Nishio \\ The University of Tokushima \\ Department of Electrical and Electronic \\ Engineering \\ Tokushima 770-8506, Japan
}

\begin{abstract}
This paper presents real polynomial form of multiple signal characterization (MUSIC) with uniform linear array (ULA). Firstly, the proposed method can reduce the computational burden of spectral music by taking the place of a large number of search points by several real roots of polynomial. Secondly, to transform Root-MUSIC algorithm into polynomial with real coefficients, a higher order Root-MUSIC whose variable is only defined on the unit upper semicircle is presented. Furthermore, this paper also improves the results obtained by J.Selva in 2005.
\end{abstract}

Index Terms - Parameter estimation, uniform linear array (ULA), array signal processing

\section{INTRODUCTION}

The multiple signal characterization (MUSIC) algorithm for direction finding [1] has been advanced for many years. To a certain degree, the more search points one use, the more accurate results one will obtain. The main computational burden locate the process of spectral search. Thus, many efficient methods are proposed to reduce the search complexity of spectral music.

A called C-MUSIC recently appears in [2]. The approach firstly divided the total interested observation field into several equal segments. The virtual source positions can be generated by spectral search over one segment and the final estimation values will be selected from these candidates. It shows from Figs. 4 and 5 of [2] that the smaller number of segments may result in the better estimation quality. However, it means that C-MUSIC needs more search points to achieve a certain accuracy.

To avoid the tremendous computation load of spectral search, Root-MUSIC [3],[4] is proposed. The search-free method has an improved resolution threshold. Taking advantage of conformal transformation, the author of [5] translated the Root-MUSIC into an univariate polynomial with real

\footnotetext{
* Xiang Cao is also with the Department of Electrical and Electronic Engineering, The University of Tokushima,Tokushima 770-8506, Japan
}

coefficients. Here, we have to point out that the interested spatial frequency in Root-MUSIC lies in the interval $[-\pi, \pi]$ instead of $[0, \pi]$. Since only half of field-of-view is considered, the real coefficients polynomial in [5] has the same degree with standard Root-MUSIC. In this paper, we improve the result and give the real polynomial form of Root-MUSIC in $[-\pi, \pi]$. The same mapping idea also appears in [6] to estimate single target. The interested range is mapped into real line by a tangent function, while the transformation function is not monotonic within target range. That is to say, the target may not be uniquely determined from the inverse transformation.

In this paper, a polynomial form with real coefficients of spectral music is presented. Our method treats the whole range of interest in uniform linear array. The complexity of spectral music can be reduced by replacing a large number of search points by real roots belonging to $[-1,1]$. Furthermore, we propose a higher order Root-MUSIC whose variable is only defined on the unit upper semicircle. The similar Root-MUSIC then is transformed into real polynomial form. The process of solving this polynomial just involves real arithmetic [7]. Our method also can be used in unitary Root-MUSIC [8] and unitary MUSIC [9].

\section{DATA MODEL}

Assume $p$ far-field narrowband signals $\left\{s_{k}\right\}$ impinging on a Uniform linear array (ULA) with $M(M>p)$ sensors. The output vector $\boldsymbol{y}(t)$ of the array at time $t$ can be written as

$$
\boldsymbol{y}(t)=\boldsymbol{A}(\boldsymbol{\theta}) \boldsymbol{s}(t)+\boldsymbol{n}(t),
$$

where $\boldsymbol{s}(t)$ is the vector of incident signals and $\boldsymbol{n}(t)$ is the vector of additive noises. The so-called array steering matrix and steering vector have the following form:

$$
\boldsymbol{A}(\boldsymbol{\theta})=\left[\boldsymbol{a}\left(\theta_{1}\right), \boldsymbol{a}\left(\theta_{2}\right), \cdots, \boldsymbol{a}\left(\theta_{p}\right)\right]
$$

$$
\boldsymbol{a}\left(\theta_{i}\right)=\left[1, e^{j \frac{2 \pi}{\lambda} d \sin \left(\theta_{i}\right)}, \cdots, e^{j \frac{2 \pi}{\lambda}(M-1) d \sin \left(\theta_{i}\right)}\right]^{T}
$$


here, $(\cdot)^{T}$ denotes transpose operator, $d$ and $\lambda$ stand for the distance between two consecutive sensors and the identical wavelength for all signals, respectively. The restriction of Directions-of-arrival (DOAs) $\theta_{1}, \cdots, \theta_{p}$ lie in the interval $\left[-\frac{\pi}{2}, \frac{\pi}{2}\right]$ for the purpose of eliminating the ambiguity of ULA [10].

Next, we define spatial frequency $\omega_{i}=\frac{2 \pi}{\lambda} d \sin \left(\theta_{i}\right)$. Then, (3) becomes

$$
\boldsymbol{a}\left(\omega_{i}\right)=\left[1, e^{j \omega_{i}}, \cdots, e^{j(M-1) \omega_{i}}\right]^{T} .
$$

For guaranteeing the uniqueness of $\boldsymbol{a}\left(\omega_{i}\right), i=1,2, \cdots, p$, the spatial frequency $\omega_{i}$ are in $[-\pi, \pi]$ instead of $[0, \pi]$ in [5]. In other words, the author in [5] only consider the half of the DOA range of interest. Since the one-to-one correspondence between $\omega_{i}$ and $\theta_{i}$, we only focus on the problem of estimating parameters $\omega_{i}, i=1,2, \cdots, p$ in this paper.

\section{REAL POLYNOMIAL FORM OF SPECTRAL MUSIC}

Under the assumptions of the uncorrelated signals and the white Gaussian noise, the array covariance matrix can be decomposed into

$$
\boldsymbol{R}=E\left\{\boldsymbol{y}(t) \boldsymbol{y}^{H}(t)\right\}=\boldsymbol{U}_{s} \boldsymbol{\Lambda}_{s} \boldsymbol{U}_{s}^{H}+\boldsymbol{U}_{n} \boldsymbol{\Lambda}_{n} \boldsymbol{U}_{n}^{H} .
$$

It is clear that the columns of $\boldsymbol{A}(\boldsymbol{\theta})$ have the maximum projection on the signal subspace $U_{s}$ and are orthogonal to the noise subspace $\boldsymbol{U}_{n}$. With this observation, two natural estimation criterions are to find the local maxima or minima [1], viz.

$$
\hat{\boldsymbol{\omega}}=\underset{\boldsymbol{\omega}}{\arg \max } f_{1}(\omega)=\underset{\boldsymbol{\omega}}{\arg \max } \boldsymbol{a}^{H}(\omega) \boldsymbol{U}_{s} \boldsymbol{U}_{s}^{H} \boldsymbol{a}(\omega)
$$

or

$$
\hat{\boldsymbol{\omega}}=\underset{\boldsymbol{\omega}}{\arg \min } f_{2}(\omega)=\underset{\boldsymbol{\omega}}{\arg \min } \boldsymbol{a}^{H}(\omega) \boldsymbol{U}_{n} \boldsymbol{U}_{n}^{H} \boldsymbol{a}(\boldsymbol{\omega}) .
$$

The total number of complex flops in (6) is $J p(2 M+1)$ and the one in (7) is $J(M-p)(2 M+1)$ [11], where $J(J \gg$ $M>p)$ is the number of spectral search points. Nevertheless, the subspace decomposition in (5) costs $\mathcal{O}\left(M^{2} p\right)$ complex flops [12],[2]. We can conclude that the main computational load of spectral MUSIC is the search steps. Consequently, it is necessary to reduce the complexity of spectral search process.

Next, we will deduce the real polynomial form of spectral music with the help of conformal transformation. By defining a translation

$$
z=e^{j\left(\frac{\omega}{2}+\frac{\pi}{2}\right)}
$$

the real point $\omega$ belonging to the domain $[-\pi, \pi]$ can be mapped to the unique complex point $z$ belonging to the unit upper semicircle. Furthermore, let us introduce a conformal transformation

$$
u=-j \frac{z-j}{z+j} .
$$

The above function is a one-to-one mapping that takes the unit upper semicircle to the interval $[-1,1]$ of real line [5],[6],[13]. Substituting (8) into (9), we get the relationship between $\omega \in[-\pi, \pi]$ and $u \in[-1,1]$ as follows:

$$
u=\tan \left(\frac{\omega}{4}\right) \text {. }
$$

This is a monotonic function in $[-\pi, \pi]$ and its range is $[-1,1]$. Thus, once the variable $u$ is estimated, the unique spatial frequency $\omega$ can be obtained by $\omega=4 \arctan u$. For this purpose, the array vector response $\boldsymbol{a}(\omega)$ must be connected to $u$.

Using $z=-j \frac{u-j}{u+j}$ and $e^{j \omega}=-z^{2}$ ( the two equations can be easily obtained from (8) and (9)), we get

$$
\begin{aligned}
{[\boldsymbol{a}(\omega)]_{m} } & =e^{j(m-1) \omega}=(-1)^{m-1} z^{2(m-1)} \\
& =\left(\frac{u-j}{u+j}\right)^{2(m-1)}, m=1,2, \cdots, M .
\end{aligned}
$$

Multiplying by $(u+j)^{2(M-1)}$ (here, we note $u \neq-j$ ), the above equation then becomes

$$
\begin{aligned}
(u+j)^{2(M-1)}[\boldsymbol{a}(\omega)]_{m} & =(u-j)^{2(m-1)}(u+j)^{2(M-m)} \\
& =c_{m, 2 M-2} u^{2 M-2}+\cdots+c_{m, 0} \\
& =\boldsymbol{c}_{m}^{T} \boldsymbol{v}(u), m=1,2, \cdots, M \quad(12)
\end{aligned}
$$

where the $(2 M-1) \times 1$ coefficient vector $\boldsymbol{c}_{m}=\left[c_{m, 0}, c_{m, 1}\right.$, $\left.\cdots, c_{m, 2 M-2}\right]^{T}$ can be calculated by convolution operation [6],[14], $\boldsymbol{v}(u)=\left[1, u, \cdots, u^{2 M-2}\right]^{T}$ is unknown real vector. Let us define an $M \times(2 M-1)$ matrix $C=$ $\left[\boldsymbol{c}_{1}, \boldsymbol{c}_{2}, \cdots, \boldsymbol{c}_{M}\right]^{T}$, the array steering vector $\boldsymbol{a}(\omega)$ can be expressed

$$
\boldsymbol{a}(\omega)=(u+j)^{2(1-M)} \boldsymbol{C v}(u)
$$

Meanwhile,a function's differential in its local extremum is zero. We can obtain [5]

$$
\frac{\partial f_{1}(\omega)}{\partial \omega}=2 \operatorname{Re}\left\{\boldsymbol{a}^{H}(\omega) \boldsymbol{W} \boldsymbol{U}_{s} \boldsymbol{U}_{s}^{H} \boldsymbol{a}(\omega)\right\}=0,
$$

where $\operatorname{Re}\{\}$ is a real operator and diagonal matrix $\boldsymbol{W}=$ $\operatorname{diag}[0, j, \cdots, j(M-1)]$. Applying (13),(14), $\boldsymbol{v}^{H}(u)=$ $\boldsymbol{v}^{T}(u)$ and dropping the scalar gives

$$
\boldsymbol{v}^{T}(u) \operatorname{Re}\left\{\boldsymbol{C}^{H} \boldsymbol{W} \boldsymbol{U}_{s} \boldsymbol{U}_{s}^{H} \boldsymbol{C}\right\} \boldsymbol{v}(u)=0 .
$$

This is an univariate polynomial of degree $4(M-1)$ with real coefficients. A similar polynomial with degree $2(M-1)$ was also presented in [5] because the author only consider $\omega \in$ 
$[0, \pi]$. However, our interesting targets are in $\omega \in[-\pi, \pi]$ [10].

Observing the equation (15), we can find that the function is similar to the Root-MUSIC in [3]. The polynomial in Root-MUSIC is $2(M-1)$-degree complex coefficients and $2(M-1)$ complex roots have to be solved in complex domain. Nevertheless in (15), we only need to calculate the real roots belonging to $[-1,1]$ in real domain. For real polynomial, the process of solution can avoid all complex arithmetic using Bairstow's method [7]. In practice, finding roots of a function usually involve iteration. The operation in (15) is $\mathcal{O}\left(16 M^{2}\right)$ real flops per iteration, while the Root-MUSIC requires $\mathcal{O}\left(24 M^{2}\right.$ ) real flops per iteration ( 1 complex flop $=6$ real flops) even though polynomial have degree $2(M-1)$.

\section{REAL POLYNOMIAL BASED ROOT-MUSIC}

In ULA, the Root-MUSIC always has more resolution compared with spectral MUSIC [4]. Thus, it is interesting to look for a real polynomial form of Root-MUSIC. Using $z=e^{j \omega}$, $\omega \in[0, \pi]$ and (9), a real polynomial with degree $2(M-1)$ has been obtained in [5]. If we consider the whole domain $\omega \in[-\pi, \pi]$, the conformal transformation equation (9) will not exist in $z=e^{j \omega}$, but exists in (8).

Thus, we can define the $m$ th element of vector $\boldsymbol{a}(z)$ as follows:

$$
[\boldsymbol{a}(z)]_{m}=(-1)^{m-1} z^{2(m-1)}, m=1,2, \cdots, M .
$$

The function $f_{2}(\omega)$ in (7) then becomes

$$
f_{2}(z)=\boldsymbol{a}^{H}(z) \boldsymbol{U}_{n} \boldsymbol{U}_{n}^{H} \boldsymbol{a}(z)=0 .
$$

This is a polynomial of degree $4(M-1)$ with complex coefficients and the variable $z$ is defined on the unit upper semicircle instead of the entire unit circle. Recalling (11)-(15) and using $\boldsymbol{a}(z)=(u+j)^{2(1-M)} \boldsymbol{C} \boldsymbol{v}(u)$, we get

$$
\boldsymbol{v}^{T}(u) \boldsymbol{C}^{H} \boldsymbol{U}_{n} \boldsymbol{U}_{n}^{H} \boldsymbol{C} \boldsymbol{v}(u)=0 .
$$

The roots of polynomial in (17) appear in reciprocal pairs $\left(z, \frac{1}{z^{*}}\right)[10]$ and could not be on the unit circle because of the effects of noise. The pair roots are related to $u$ by conformal transformation (9),

$$
u_{1}=-j \frac{z-j}{z+j}, \quad u_{2}=-j \frac{1-j z^{*}}{1+j z^{*}} .
$$

Obviously, we have $u_{1}=u_{2}^{*}$. It is reasonable to conclude that the roots in (18) also appear in conjugate pairs like $\left(u, u^{*}\right)$. As a consequence, the equation (18) is a real coefficients polynomial, namely

$$
\boldsymbol{v}^{T}(u) \operatorname{Re}\left\{\boldsymbol{C}^{H} \boldsymbol{U}_{n} \boldsymbol{U}_{n}^{H} \boldsymbol{C}\right\} \boldsymbol{v}(u)=0 .
$$

Next, we will analyze how to estimate the spatial frequency $\omega_{i}, i=1,2, \cdots, p$ from the conjugate roots $\left(u_{i}, u_{i}^{*}\right)$,

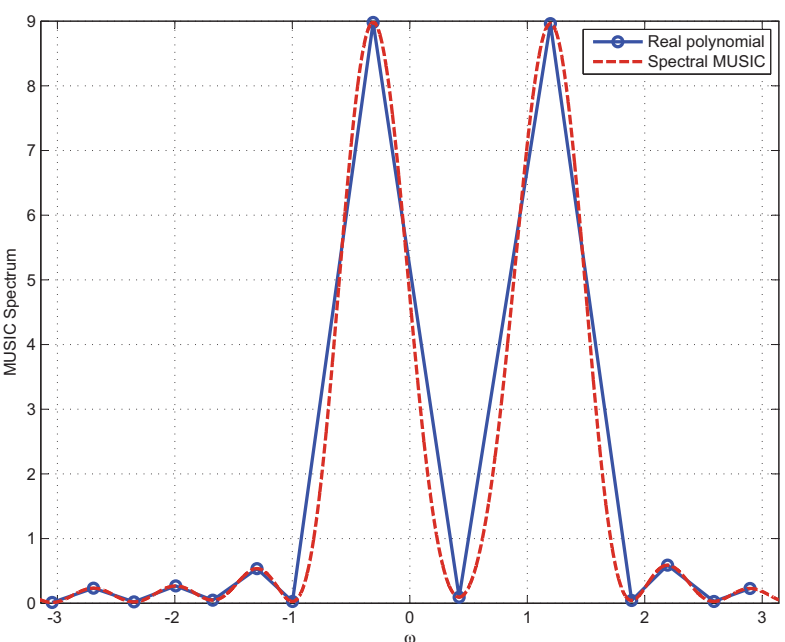

Fig. 1. MUSIC spectrum with 1000 points and Real polynomial spectrum with 14 points. The number of snapshots $N=100, \mathrm{SNR}=5 \mathrm{~dB}$

$i=1,2, \cdots, 2(M-1)$ of above polynomial. Assuming $z=a+j b, b \geq 0$, the equation (9) becomes

$$
u=-j \frac{a+j b-j}{a+j b+j}=\frac{-2 a-j\left(a^{2}+b^{2}-1\right)}{a^{2}+(b+1)^{2}} .
$$

When the roots $z$ in (17) are close to the unit circle, the imaginary part of $u$ must tend to zero. Therefore, $p$ real parts of $u_{i}$ can be picked up through comparing the absolution value of imaginary part of $u_{i}, i=1,2, \cdots, 2(M-1)$. The spatial frequency will be given by $\omega_{i}=4 \arctan \left[\operatorname{Re}\left(u_{i}\right)\right], i=$ $1,2, \cdots, p$.

Note that, although the polynomial in (20) have $4(M-1)$ degree, its coefficients are real and roots appear in conjugate pairs. Thus, we only need to solve $2(M-1)$ roots. The whole process just involve real arithmetic.

\section{EXPERIMENTS}

In this section, two examples were conducted by computer simulation. In all scenarios, two equal-power uncorrelated signals are considered and a ULA have 9 sensors. Meanwhile, we set spatial frequency $\omega_{1}=-0.3, \omega_{2}=1.2$. Here, $\omega_{1}$ can not be solved in [5].

Example 1: Performance of real polynomial based on spectral MUSIC

We firstly should calculate $\omega_{i}=4 \arctan u_{i}, i=$ $1,2, \ldots, Q\left(u_{i} \in[-1,1]\right.$ can be obtained by solving the real polynomial (15)). Then, evaluating the extremum of (6) at $\omega_{i}, i=1,2, \cdots, Q$ can pick up the final value $\hat{\omega}_{k}, k=1,2, \cdots, p$. The computational complexity of the above process is $\mathcal{O}\left(8 M^{2}+16 Q M^{2}\right)+6 Q p(2 M+1)$ real flops [6]. 


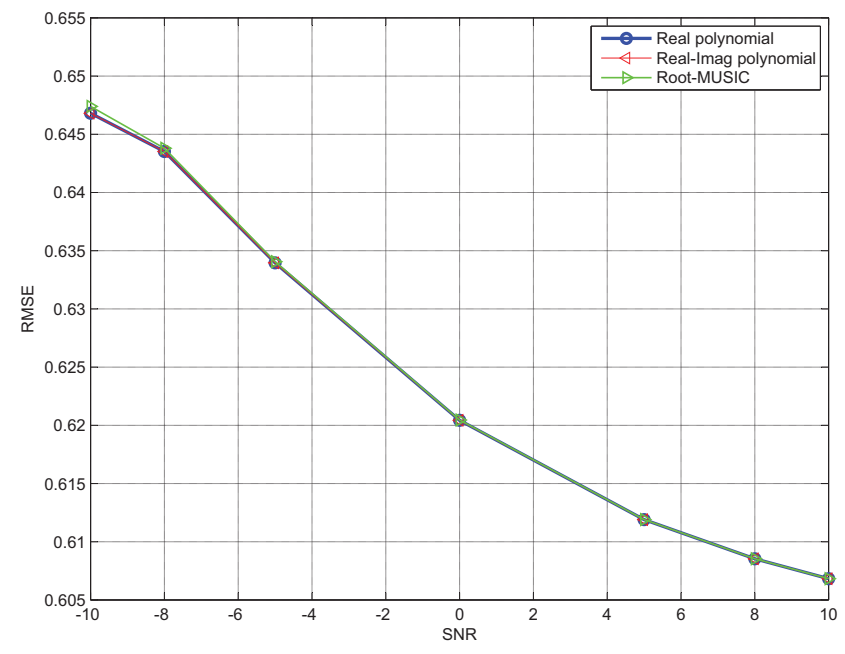

Fig. 2. RMSE of spatial frequency $\omega=-0.3$. The number of snapshots $N=100$

In this example, 14 real roots of the polynomial (15) lie in the domain $[-1,1]$. Figure. 1 shows that the proposed method can capture the extremal point. In addition, our method requires about 21984 real flops, while the spectral MUSIC requires 36000 complex flops. In other words, the computation load of real polynomial algorithm is roughly equal to the spectral MUSIC with 100 search points.

Example 2: Performance of real polynomial based on Root-MUSIC

In the second case, we mainly test the validity of real coefficients polynomial (20). For this purpose, let us introduce the imaginary part of matrix $\mathcal{H}=\boldsymbol{C}^{H} \boldsymbol{U}_{n} \boldsymbol{U}_{n}^{H} \boldsymbol{C}$. After simple derivation, we obtain

$$
\boldsymbol{v}^{T}(u)\{\operatorname{Re}\{\mathcal{H}\}+\operatorname{Im}\{\mathcal{H}\}\} \boldsymbol{v}(u)=0 .
$$

This approach using (22) named real-imag polynomial and was indicated by red line in Figure. 2. It is worth pointing out that (20) and (22) give the same results. That is to say, the coefficients of polynomial (18) are real. Meanwhile, the behavior of our method have been improved a little at lower signal-to-noise ratio (SNR) than Root-MUSIC [3].

\section{CONCLUSIONS}

An univariate polynomial with real coefficients is presented for reducing the search points in spectral MUSIC. The real roots belonging to $[-1,1]$ only need to be solved and the process of solution can avoid all complex arithmetic. On the other hand, for improving the results in [5], we propose a similar Root-MUSIC whose variable is defined on the unit upper semicircle. Then, the complex equation is also transformed into real polynomial form.

\section{ACKNOWLEDGMENT}

This work was supported in part by the National Natural Science Foundation of China under Grant 61172162, the State Key Development Program for Basic Research of China under Grant 2010CB327902 and the Suzhou Natural Science Foundation under Grant SYG201224.

\section{REFERENCES}

[1] R. O. Schmidt, "Multiple emitter location and signal parameter estimation," IEEE Trans. Antennas Propag., vol. 53, pp. 276-280, Mar. 1986.

[2] F. G. Yan, M. Jin, and X. Qao, "Low-complexity doa estimation based on compressed music and its performance analysis," IEEE Trans. Signal Process., vol. 61, pp. 1915-1930, Apr. 2013

[3] A. J. Barabell, "Improving the resolution performance of eigenstructure-based direction-finding algorithms," in Proc. ICASSP, Boston, MA, May 1983, pp. 336-339.

[4] B. D. Rao and K. V. S. Hari, "Performance analysis of root-music," IEEE Trans. Acoust.,Speech, Signal Process., vol. 37, pp. 1939-1949, Dec. 1989.

[5] J. Selva, "Computation of spectral and root music through real polynomial rooting," IEEE Trans. Signal Process., vol. 53, pp. 1923-1927, May 2005.

[6] J. X. Wu, T. Wang, and Z. Bao, "Fast realization of maximum likelihood angle estimation with small adaptive uniform linear array," IEEE Trans. Antennas Propag., vol. 58, pp. 3951-3960, Dec. 2010.

[7] W. H. Press, S. A. Teukolsky, W. T. Vetterling, and B. P. Flannery, Numerical recipes in $C$, Cambridge Univ. Press, Cambridge, U.K., 1997.

[8] M. Pesavento, A. B. Gershman, and M. Haardt, "Unitary root-music with a real-valued eigendecomposition: a theoretical and experimental performance study," IEEE Trans. Signal Process., vol. 48, pp. 1306-1314, May 2000.

[9] K. C. Huarng and C. C. Yeh, "A unitary transformation method for angle-of-arrival estimation," IEEE Trans. signal process., vol. 39, pp. 975-977, Apr. 1991.

[10] P. Stoica and R. L. Moses, Spectral analysis of signals, Prentice-Hall, 2005.

[11] D. S. Watkins, Fundamentals of matrix computations, Wiley, 2004.

[12] Y. Zhang and B. P. Ng, "Music-like doa estimation without estimating the number of sources," IEEE Trans. Signal Process., vol. 58, pp. 1668-1676, Mar. 2010. 
[13] L. Blanco, J. Serra, and M. Nájar, "Conformal transformation for efficient root-toa estimation," in Proc. ISCCSP, Malta, Mar. 2008, pp. 1314-1319.

[14] M. Costa and V. Koivunen, "Incorporating array nonidealities into adaptive capon beamformer for source tracking," in Proc. SAM, Hoboken, NJ, Jun. 2012, pp. 445448. 